\title{
EMPLOI ET SIGNIFICATION \\ DES CULTURES DE DERMATOPHYTES SUR TERRE ET MILIEUX A BASE DE TERRE
}

\author{
Par R. VANBREUSEGHEM(') et M. VAN BRUSSEL
}

Les Dermatophytes sont des champignons pathogènes, apparentés aux Gymnoascales. Leur étude esquissée, il y a cent ans, par Schoenlein, Remak et Gruby, se développa pour attẹindre sa pleine efflorescence au début de ce siècle, grâce aux travaux de R. Sabouraud et de E. Bodin. L'ampleur donnée par Sabouraud à l'expression de ses observations dans son remarquable Précis des Teignes (1910) fut telle qu'on le considère à juste titre comme le fondateur de cette branche particulière de la médecine et de la parasitologie qui s'occupe des dermatophyties. Il faut comprendre sous ce terme les maladies causées par des Dermatophytes (Vanbreuseghem 1951) et l'employer de préférence aux vocables anciens : teigne, eczéma marginé, herpès circiné, athlete's foot.

Nous pensons exprimer correctement l'opinion générale qui prévaut depuis Sabouraud, en disant que les Dermatophytes ont été considérés jusqu'ici comme des parasites exclusifs de l'homme et des animaux, chez lesquels ils déterminent des épidémies et des épizooties d'allure aiguë ou chronique. I. Muende et P. Webb (1937) furent probablement les premiers à leur attribuer une vie saprophytique propre, en décrivant et isolant de crottin de cheval un Dermatophyte responsable d'une épidémie de teigne survenue chez des veaux et des chevaux. Cependant, des études importantes, faites dans le but d'isoler des Dermatophytes du milieu extérieur avaient abouti à un échec (L. Bonar et A.-D. Dryer 1932, J.-W Williams, 1933-1934).

En novembre 1951 C. W. Emmons et, en janvier 1952, indépendamment de cet auteur, Vanbreuseghem et Van Brussel réussirent à cultiver des Dermatophytes sur de la terre de jardin stérilisée. Emmons se bornait à signaler la culture de Trichophyton menta-

(1) Etude effectuée avec l'aide et un subside de l'Institut pour la Recherche Scientifique en Afrique Centrale (I.R.S.A.C.).

Ann. de Parasitologie, $\mathrm{T}$. XXXII, No $5 .-1952$. 
grophytes, tout en incluant ce fait dans l'ensemble de l'épidémiologie des mycoses. Vanbreuseghem (1952), presque simultanément, exprimait ses vues sur ce qu'il appelait le cycle biologique des Dermatophytes et, se servant des mêmes arguments que Emmons, exprimait une vue d'ensemble sur le stade saprophytique des champignons pathogènes. D'autre part, Vanbreuseghem et Van Brussel (1952) venaient de faire leurs premières observations sur l'emploi des milieux additionnés de terre pour la culture des Dermatophytes. Une étude attentive de la littérature permit de constater que, dans de rares cas, un Dermatophyte, - Microsporum gypseum -, avait été isolé du sol (Cfr. G.-R. Mandels, W.-H. Stahl et H.-S. Levinson, 1948, W.-L. White, G.-R. Mandels et R.-G. Siu, 1952, M. Gordon, 1952, suivant une communication personnelle de L. Ajello). Ces faits, sur la valeur desquels il convient toutefois de se montrer quelque peu circonspect, appuieraient de leur poids les opinions exprimées indépendamment par Emmons et Vanbreuseghem.

Le but du présent travail est de faire un exposé complet des observations que nous avons faites jusqu'à ce jour dans ce domaine essentiellement nouveau.

\section{I. - Milieux utilisés}

a) Milieu de Sabouraud :

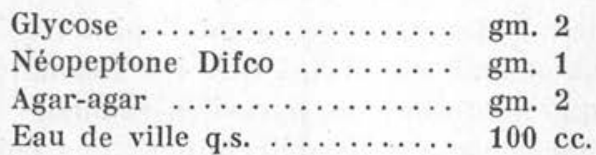

b) Sabouraud-terre :

\begin{tabular}{|c|c|}
\hline Glycose $\ldots \ldots \ldots \ldots \ldots$ & gm. 2 \\
\hline Néopeptone Difco ...... & gm. 1 \\
\hline Agar-agar $\ldots \ldots \ldots \ldots \ldots$ & gm. 2 \\
\hline Terre .... & $\mathrm{gm} .20$ \\
\hline ville q.s. . . . & $100 \mathrm{cc}$ \\
\hline
\end{tabular}

c) Conservation-terre :

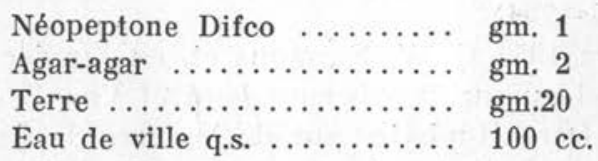

d) Gélose-terre :

Agar-agar ............. gm. 2

Terre ................ gm.20

Eau de ville q.s. ......... $100 \mathrm{cc}$. 
Ces milieux sont préparés de la manière suivante : l'agar-agar est placé dans une quantité d'eau suffisante pour le faire gonfler. Le glycose, la peptone, la terre sont ajoutés, puis on verse de l'eau jusqu'au volume désiré. On stérilise 30 minutes à $115^{\circ}$ à l'autoclave. On répartit en tubes à essai. On procède à une nouvelle stérilisation durant 30 minutes à $115^{\circ}$ et on incline les tubes. Ceux-ci sont en pyrex et mesurent $220 \times 20 \mathrm{~mm}$. Le milieu de culture a un $\mathrm{pH}$ d'environ 6-6,5.

La terre est une terre de jardin riche en humus. Avant de l'ajouter aux différents milieux, on la tamise sur un fin tamis pour éliminer les débris végétaux et autres. Les milieux renfermant de la terre ont un $p \mathrm{H}$ à peu près égal à celui du milieu de Sabouraud.

e) Terre à l'état brut :

La terre tamisée est répartie dans des boîtes de Pétri ou dans des anneaux de verre fixés sur des lames porte-objets avec du lut de RondeauDunoyer. Dans ce dernier cas, les petites cuvettes ainsi constituées sont placées dans une boîte de Pétri dont le fond' est inondé d'eau stérile. Le lut de Rondeau-Dunoyer ne résiste pas extrêmement longtemps à l'humidité, mais ce produit nous a cependant donné satisfaction.

La terre est stérilisée par autoclavage à $115^{\circ}$ durant 30 minutes.

\section{II. - Cultures de Dermatophytes sur milieux à base de terre}

Nos observations ont porté sur les espèces suivantes : Ctenomyces (Trichophyton) interdigitalis, Sabouraudites (Microsporum) canis, Trichophyton rubrum, Langeronia (Trichophyton) soudanensis, Epidermophyton floccosum. Les ensemencements sont pratiqués comme d'habitude en un point du milieu de culture et les tubes sont maintenus à $25^{\circ} \mathrm{C}$, durant toute la durée de l'expérience.

\section{a) Ctenomyces interdigitalis}

Sur Sabouraud, cette souche présente sa morphologie normale : colonie poudreuse, blanchâtre, légèrement brunâtre, vers le centre. Sur Sabouraud-terre, elle prend à peu près le même aspect, mais la teinte brunâtre est un peu plus accusée. Sur conservation-terre, aspect semblable à ce qu'il est sur Sabouraud-terre, mais développement moindre. Sur gélose-terre, l'aspect est absolument différent : c'est un fin duvet blanc qui court à la surface de la gélose. Il est peu dense et ne forme pas de colonies consistantes. Ctenomyces interdigitalis sera l'espèce qui, sur gélose-terre, se développera le plus aisément.

L'examen microscopique des cultures sur Sabouraud sont typiques : aleuries très abondantes, rondes, disposées surtout en grap- 
pes. Au début, il n'y a ni fuseaux, ni images en bois de cerf, ni hyphes en crosse, ni spires. Sur Sabouraud-terre, aleuries très nombreuses, hyphes en crosses et spires, mais pas de fuseaux. Sur conservation-terre, aleuries, hyphes en crosses, spires, pas de fuseaux. Sur gélose-terre, aleuries, hyphes en crosse, bois de cerf, spires et nombreux fuseaux.

\section{b) Sabouraudites canis}

Les colonies sur Sabouraud sont jaunâtres, duveteuses, et renferment de nombreux fuseaux. Au début on ne trouve pas d'aleuries. Sur Sabouraud-terre, les colonies sont beaucoup plus blanches et le diagnostic de $S$. canis est difficile : mais l'examen microscopique montre des fuseaux et des aleuries. Sur conservation-terre, aspect semblable à ce qu'il est sur Sabouraud-terre. Sur géloseterre, le développement est très restreint et nécessite l'usage de la loupe pour être bien observé : ce sont des filaments disposés en réseau à la surface de la gélose. Cependant, il y a de nombreux fuseaux. Sur tous les milieux additionnés de terre, les fuseaux de $S$. canis sont énormes, atteignant $75 \mu$ de long pour 16 à $18 \mu$ de large. D'autres sont beaucoup plus longs encore $(180 \mu)$, mais beaucoup moins larges $(2,5$ à $4 \mu)$.

\section{c) Trichophyton rubrum}

Sur Sabouraud, cette souche a son aspect le plus habituel : duvet blanc sur un beau rouge vineux ; microscopiquement, aleuries piriformes, disposées suivant le type Acladium, ou en petites grappes. L'aspect sur Sabouraud-terre est fort semblable, tant macroscopiquement que microscopiquement. Sur conservation-terre, la colonie ne forme pas de pigment et tend à devenir poudreuse ; les aleuries sont innombrables. Sur gélose-terre, le développement est pauvre, meilleur que pour $S$. canis, mais bien moins abondant que celui de $C t$. interdigitalis. L'examen microscopique ne révèle que des aleuries, disposées suivant le type Acladium, et de très nombreuses chlamydospores, disposées surtout en chaînettes.

\section{d) Langeronia soudanensis}

Sur Sabouraud, colonie acuminée de teinte rouille, se continuant sur la gélose par des rayons de filaments; l'aspect microscopique est celui décrit par Vanbreuseghem à l'occasion de la création du 
genre Langeronia (1950): hyphes secondaires se dirigeant dans le sens opposé à la croissance générale, aspect buissonneux, arthrospores, très rares aleuries. Sur Sabouraud-terre, le développement est excellent, et semblable à ce qu'il est sur Sabouraud, mais vers le vingt-cinquième jour trois colonies sur six développent un mutant violet (Cfr. infra). Un peu plus tard, toutes les colonies muteront. L'aspect microscopique de la partie mutée est à peu près identique à ce qu'elle est sur Sabouraud : il n'y a pas ou péu d'aleuries (Cfr. cependant infra). Sur conservation-terre, le développement est pauvre mais rappelle toujours l'aspect des colonies sur Sabouraud: les filaments mycéliens ont la morphologie décrite ci-dessus, et il n'y a pas ou peu d'aleuries. Sur gélose-terre, le développement est très restreint, et se présente sous l'aspect d'un petit disque blanchâtre, dans lequel l'examen microscopique révèle de très nombreuses aleuries, disposées suivant le type Acladium, des chlamydospores nombreuses; les filaments mycéliens sont moins typiques que sur les autres milieux, les caractères morphologiques du genre Langeronia restant cependant très accusés.

\section{e) Epidermophyton floccosum}

Sur Sabouraud, c'est le classique disque jaune-verdâtre poudreux et légèrement plissé, aux innombrables fuseaux disposés en régime de banane. Sur Sabouraud-terre, la culture est presque blanche, légèrement duveteuse, et renferme de très nombreux fuseaux. Sur conservation-terre, les colonies ont les mêmes caractères que sur Sabouraud-terre. Sur gélose-terre, le développement est très pauvre, plus pauvre encore peut-être que celui de $L$. soudanensis : à la loupe, on peut observer des filaments courant à la surface de la gélose, et réunis en réseau par quelques points un peu plus marqués. L'examen microscopique montre des fuseaux et des vrilles, ce qui n'a été qu'exceptionnellement rencontré par d'autres auteurs.

Nous n'avons pas insisté sur les caractères des filaments mycéliens sur les milieux à base de terre. On sait que si les filaments mycéliens des dermatophytes sont généralement riches en protoplasme dans les primo-cultures, celui-ci diminue considérablement dans les sub-cultures. Il en est de même d'ailleurs des formes de reproduction : aleuries et fuseaux, qui, dans les primo-cultures, sont gonflées de protoplasme, tandis que dans les sub-cultures elles s'appauvrissent en cette substance. Or, il suffit de faire pousser un dermatophyte sur milieux à base de terre, pour assister 
aussitôt à une recharge en protoplasme du mycélium et des formes de reproduction. C'est pour les Dermatophytes une véritable fontaine de Jouvence, qui conduit à leur rajeunissement. Les colonies ainsi obtenues sont particulièrement vivaces, même sur géloseterre, ainsi que le démontrent les sub-cultures parfaitement jeunes, obtenues après six mois de séjour des Dermatophytes sur ce milieu. En conclusion, nous disons :

$1^{\circ}$ que les Dermatophytes se développent bien sur des milieux à base de terre ;

$2^{\circ}$ que la production des formes de reproduction est plus abondante sur ces milieux que sur Sabouraud;

. $3^{\circ}$ qu'ils y subissent un phénomène de rajeunissement, caractérisé par la richesse en protoplasme du mycélium, des microconidies et des macroconidies.

\section{III. - Culture des Dermatophytes sur terre}

Nous avons eu recours à deux méthodes pour ensemencer les Dermatophytes sur terre stérilisée en boîte de Pétri :

a) Ensemencement direct : On prélève un petit fragment de colonie qu'on dépose sur la surface de la terre stérile. Le développement restreint auquel aboutit cette méthode d'ensemencement nous l'a fait abandonner au bénéfice de la méthode suivante :

b) Ensemencement par émulsion : On émulsionne dans de l'eau physiologique la surface d'une colonie de dermatophyte. 10 cc. d'eau physiologique conviennent pour une colonie normalement obtenue sur milieu de Sabouraud. Environ 3 cc. de cette émulsion sont répartis aussi également que possible à la surface de la terre stérile.

La mise en évidence du développement du Dermatophyte n'est pas aisée par les méthodes ordinaires. L'observation directe, à l'œil nu, ne montre, au mieux, lorsque le développement est abondant, qu'un aspect velouté de la surface de la terre. L'examen microscopique entre lame et lamelle dans le lugol est rendu difficile par les particules de terre qui s'opposent au contact des deux surfaces de verre et on ne réussit à voir, après de patientes recherches, que des fragments de mycélium ou quelques formes de reproduction, le plus souvent adhérentes à des particules de terre. Deux techniques d'observation nous ont paru dignes d'être retenues. La première, c'est l'examen des cultures en lumière de Wood: les colonies de Dermatophytes développées à la surface de la terre 
montrent une légère fluorescence blanche, qui contraste avec la couleur violette que la terre prend sous l'influence de la lumière de Wood. La deuxième technique d'observation consiste dans l'emploi d'une loupe binoculaire pour dissections : on place la boite de Pétri renfermant la terre sous l'objectif et, avec un bon éclairage, on observe aisément le développement de Dermatophytes à la surface de la terre. Ce développement est abondant et consiste en un réseau de filaments mycéliens supportant des formes de reproduction qui, lorsqu'elles sont assez grandes, comme les macroconidies du $S$. canis, sont aisées à reconnaître.

Nous pouvons conclure de cette série d'expérience que les Dermatophytes se développent aisément, en faisant apparaitre leurs formes de reproduction sur la terre stérile.

\section{IV. - Cultures sur terre stérile et sur milieux à base de terre de produits parasités par les Dermatophytes (cheveux, poils, squames, ongles)}

Encore que cela fût probable, il restait à démontrer que des cheveux, des poils, des squames ou des ongles parasités par des Dermatophytes pourraient donner des colonies de Dermatophytes sur terre stérile et sur milieux à base de terre. Nous avons utilisé pour nos ensemencements des produits pathologiques, dont l'examen antérieur nous avait démontré le parasitisme, et dont la culture nous avait révélé l'espèce du parasite. C'est ainsi que nous sommes partis de poils de cobayes envahis par Ctenomyces interdigitalis ; de fragments d'ongles humains parasités par Trichophyton rubrum ; de cheveux présentant une lésion microsporique, produite par Sabouraudites langeroni ou S. rivalieri; de poils de chats et de chiens infectés par $S$. canis; de cheveux présentant une lésion endothrix produite par Langeronia soudanensis. Poils, cheveux, squames, fragments d'ongles, sont simplement déposés à la surface de la terre stérile contenue dans un godet placé dans une boite de Pétri, dont le fond est inondé d'eau stérile. Le tout est maintenu à $25^{\circ} \mathrm{C}$.

La mise en culture sur milieux à base de terre de ces produits pathologiques nous a donné des colonies dont l'aspect est semblable à celui obtenu par des sub-cultures de dermatophytes sur les mêmes milieux. Nous ne nous y attarderons pas.

Les cultures sur terre stérile ont toutes été réalisées dans des godets de verre remplis de terre stérile, placés au fond d'une boîte de Pétri. Les godets sont fabriqués, comme nous l'avons dit plus 
haut, à l'aide d'un anneau de verre collé sur une lame porte-objet, avec du lut de Rondeau-Dunoyer.

Quelques centimètres cubes d'eau distillée stérile sont versés dans le fond de la boite de Pétri pour réaliser une atmosphère humide. L'étude du développement se fait à l'aide de la loupe binoculaire. On voit, dans l'espace de quelques jours, des filaments mycéliens partir du fragment de cheveu, de l'ongle, des squames ou du poil, et s'étendre à la surface de la terre, vers les bords du godet. Lorsque le Dermatophyte parasitant le produit pathologique donne des fuseaux, ceux-ci se développent à la surface de la terre. On pourrait objecter que la substance riche en kératine, qui a servi de point de départ pour l'inoculation, sert de source alimentaire pour le Dermatophyte auquel elle donne naissance. Ceci est vrai en partie, mais on peut aisément démontrer que la terre seule peut permettre ce développement. Il suffit, par exemple, dans le cas du $S$. canis, où l'observation est particulièrement aisée à cause de la grande abondance des fuseaux, d'enlever après quelques jours, dès que le développement du mycélium a commencé de se faire, le poil qui a servi d'inoculum. On peut alors constater que, malgré la soustraction de la substance kératinienne, le $S$. canis continue à se développer à la surface de la terre, jusqu'à gagner les bords du godet, bien loin du point d'ensemencement.

Ce fait démontre que des produits pathologiques, parasités par des Dermatophytes et abandonnés sur le sol, peuvent y trouver un milieu adéquat pour leur reproduction.

Les produits pathologiques dont nous sommes partis pour procéder aux inoculations avaient des âges différents. Les poils de chats et de chiens parasités par $S$. canis avaient été récoltés sur les animaux cinq et dix jours avant l'expérience ; les fragments d'ongles parasités par $T$. rubrum avaient été prélevés sur une jeune fille trois mois et demi avant que l'expérience ne soit réalisée ; les cheveux parasités par S. langeroni, par S. rivalieri et par $L$. soudanensis, avaient été récoltés au Congo belge sur des indigènes, respectivement quatre mois, six mois et six mois avant d'être soumis à l'expérimentation.

\section{V. - Pouvoir pathogène des colonies de Dermatophytes développées sur terre}

On pouvait se demander si les colonies de Dermatophytes développées sur terre conservaient leur pouvoir pathogène. Ce fait pouvait être démontré par l'inoculation expérimentale à l'homme ou au cobaye. Nous avons eu recours à ces deux méthodes : 


\section{a) Inoculation humaine expérimentale}

Nous sommes partis de poils de chien parasités par $S$. canis. Un de ces poils est inoculé sur terre stérile, suivant la technique décrite ci-dessus. Après quinze jours, le Dermatophyte s'est développé abondamment à la surface de la terre et les macroconidies lancéolées sont abondantes, logées dans de petites dépressions de la terre comme des œufs dans un nid. On prélève avec la pointe d'une aiguille stérile une minuscule partie de cette culture. Le prélèvement, qui n'est visible qu'à la loupe, est mélangé avec une petite quantité de miel. Le mélange est inoculé par scarification à la cuisse d'une jeune fille de 19 ans, parasitée depuis douze ans par Trichophyton rubrum. La patiente, revue quatorze jours après l'inoculation, montre à l'endroit inoculé un petit placard squameux large d'un centimètre de diamètre. On met en évidence dans les squames de nombreux filaments mycéliens. La culture de ces squames donne des colonies typiques de $S$. canis. Un mois plus tard, l'endroit inoculé est encore positif.

\section{b) Inoculation expérimentale au cobaye}

Dans une première série d'expériences, nous sommes partis de terre inoculée avec une émulsion de Ctenomyces interdigitalis et de Trichophyton rubrum. Les inoculations ont été pratiquées sur la peau rasée du cobaye, avec des cultures âgées de 30 jours et de 71 jours pour $C t$. interdigitalis, et de 66 jours pour $T$. rubrum. Dans les trois cas, nous avons obtenu des inoculations positives, vérifiées cliniquement, microscopiquement, et par culture. Il nous a paru que les inoculations réalisées à partir de cultures sur terre réussissaient plus facilement qu'à partir de cultures sur milieu de Sabouraud, et qu'elles duraient plus longtemps (jusque deux mois après l'inoculation).

Dans une deuxième série d'expériences, nous avons inoculé au cobaye les cultures âgées de 19 jours, obtenues à partir d'un poil de chat parasité par $S$. canis, et ensemencé sur terre stérile. Les résultats ont été aussi démonstratifs que dans la première série d'expériences.

On peut conclure de tout ceci que les Dermatophytes développés sur terre, soit à partir de cultures, soit à partir de produits pathologiques, conservent leur pouvoir pathogène pour l'homme et les animaux et que, vraisemblablement, leur pouvoir pathogène est accru par rapport à celui des colonies de Dermatophytes développées sur les milieux de culture usuels. 


\section{VI. - Réversibilité du pléomorphisme}

Le pléomorphisme, considéré comme une dégénérescence irréversible des Dermatophytes entretenus sur les milieux usuels de laboratoire, en particulier sur les milieux sucrés, est le plus grand ennemi des collections de Dermatophytes : avec son apparition coïncide la disparition des espèces types ; à cause de lui, il existe de grosses difficultés dans la classification des Dermatophytes. Le pléomorphisme consiste essentiellement en la disparition des formes de reproduction, aleuries et fuseaux, et dans l'appauvrissement progressif des filaments mycẻliens en protoplasme. Or, l'emploi des milieux à base de terre nous a permis de constater que ceux-ci favorisaient l'apparition des formes de reproduction et provoquaient un enrichissement des filaments en protoplasme. Cela nous conduisit à étudier le développement sur les milieux à base de terre de deux souches pléomorphiques de Dermatophytes. L'une d'entre elles est un Ctenomyces persicolor, isolé par l'un d'entre nous en 1949 (Vanbreuseghem, 1949). La souche initiale était représentée par des colonies poudreuses, couleur pêche, extrêmement riches en formes de reproduction. Ces caractères ont pu être maintenus pendant un an par des repiquages réguliers sur milieu de Sabouraud et milieu de conservation, mais on a assisté progressivement à la disparition des formes de reproduction, à l'amincissement des filaments et à leur appauvrissement en protoplasme : actuellement, la souche est complètement blanche, duveteuse, c'est une souche pléomorphique des plus typiques. Cette souche pléomorphique, ensemencée en novembre 1951 sur Sabouraud ou sur milieu de conservation, ne donnait qu'un duvet blanc absolument stérile. Sur gélose-terre, elle se développe sous l'aspect d'un duvet peu abondant, mais l'examen microscopique révèle très rapidement de fort nombreuses microconidies rondes, disposées suivant le type Acladium ou en grappes. Sur conservation-terre, treize jours après l'inoculation, nous constatons, dans la partie de la colonie orientée vers le fond du tube, une zone violacée, poudreuse, qui renferme de très nombreuses microconidies. L'aspect de cette partie n'est pas sans rappeler étroitement la souche à ses débuts. De cette partie violacée et poudreuse, nous faisons des ensemencements sur Sabouraud et sur conservation-terre; ultérieurement, nous ferons des passages sur Sabouraud-terre. Sur conservationterre, après plusieurs passages, nous finissons par obtenir une souche, qui rappelle à s'y méprendre la primo-culture de Ctenomyces 
persicolor, que nous avons isolée en 1949. Sur les autres milieux, nous obtenons toujours une souche pléomorphique, même sur Sabouraud-terre, encore que l'on trouve dans la culture d'assez nombreuses microconidies. Ces différents essais, que nous ne pouvons exposer dans tous leurs détails, nous montrent qu'il est possible de déterminer le retour à la normale d'un Dermatophyte en partant d'une souche pléomorphisée, par passage sur milieu à base de terre dépourvu de sucre. Depuis six mois, nous avons entretenu sur conservation-terre cette souche redevenue normale de Ct. persicolor: elle l'est restée. Pendant cette même période de temps, la souche pléomorphique entretenue sur Sabouraud est restée tout à fait pléomorphique. Notons encore que sur conservationterre nous avons vu apparaître de très nombreux fuseaux.

Un autre exemple de réversibilité du pléomorphisme à la normale nous a été donné par une souche d'Epidermophyton floccosum, isolée en mars 1949. Comme il est courant d'en faire l'observation avec cette espèce de Dermatophyte, la souche s'est très rapidement pléomorphisée, et nous ne l'avions conservée dans notre collection que parce qu'elle avait été isolée au Congo belge, où le parasite ne semble pas jusqu'ici extrêmement fréquent. Dès le premier passage sur conservation-terre, de nombreux fuseaux massués ont fait leur apparition, et l'aspect de la colonie dans sa partie inférieure, - nous voulons dire la partie orientée vers le fond du tube, celui-ci étant debout -, a pris un aspect normal. Un repiquage sur conservation-terre nous a donné une colonie normale. La même colonie a été obtenue sur Sabouraud-terre, ce qui n'est pas le cas pour $C t$. persicolor, qui exige l'absence de sucre pour conserver ses caractères normaux.

Eneouragés par ces résultats, nous avons tenté d'en obtenir de plus convaincants encore en partant, de souches pléomorphisées depuis des dizaines d'années : jusqu'ici nos essais ont été infructueux.

Quoi qu'il en soit, les observations que nous avons faites nous obligent à modifier nos points de vue habituels sur le pléomorphisme. Il n'est plus possible de considérer ce phénomène comme un phénomène de dégénérescence irréversible. Peut-être cela est-il vrai pour des souches extrêmement vieilles, mais ce n'est certainement pas toujours le cas. Nous sommes en tout cas en possession d'une méthode qui nous donne l'espoir de rafraîchir nos collections, plus ou moins altérées, et de rendre quelque vivacité aux couleurs, qui semblaient définitivement disparues. 
Les observations que nous venons de rapporter nous ont conduits tout naturellement à nous demander s'il n'était pas possible de se servir des milieux à base de terre pour conserver les Dermatophytes. On sait que, outre la méthode classique, qui consiste à effectuer des repiquages mensuels sur le milieu de conservation, sont venues s'ajouter, assez récemment, deux méthodes nouvelles. L'une d'entre elles, la lyophilisation, n'est applicable qu'aux Dermatophytes qui donnent de nombreuses formes de reproduction ; par suite de cette exigence, elle n'est applicable qu'à un petit nombre d'espèces. L'autre méthode consiste à entretenir les colonies sous huile minérale. Ce procédé permet de conserver vivantes durant près de deux ans les souches de Dermatophytes, mais malheureusement, comme l'ont très bien démontré récemment L. Ajello, V.-Q. Grant et M.-A. Gutzke (1951), il ne s'oppose pas au pléomorphisme.

Nous possédions, au moment d'entreprendre la présente série d'expériences, cinq souches de Dermatophytes maintenues depuis cinq mois, sans aucun repiquage, sur les milieux suivants : Sabouraud, Sabouraud-terre, conservation-terre et gélose-terre. Les souches de Dermatophytes conservées sur ces divers milieux étaient : Ctenomyces interdigitalis, Trichophyton rubrum, Sabouraudites canis, Epidermophyton floccosum et Langeronia soudanensis. Ces souches, au moment où elles avaient subi leur dernier ensemencement, étaient isolées respectivement depuis 15 jours, 15 jours, 210 jours, 120 jours et 15 jours. On peut distinguer parmi elles deux espèces se pléomorphisant facilement : Epidermophyton floccosum et Sabouraudites canis. Les trois autres manifestent une tendance beaucoup plus restreinte au pléomorphisme. Le même jour, chacune de ces souches fut repiquée sur milieu de Sabouraud à partir des quatre milieux différents sur lesquels elles avaient été maintenues. L'observation nous a montré : $a$ ) que toutes les souches se développaient bien sur Sabouraud, mais que celles provenant de milieux à base de terre se développaient plus rapidement ; $b$ ) que les souches de $S$. canis et de $E$. floccosum obtenues sur Sabouraud étaient pléomorphiques, sauf celles obtenues à partir de géloseterre, qui étaient parfaitement fraîches ; $c$ ) que les autres souches, $T$. rubrum, Ct. interdigitalis et L. soudanensis étaient semblables quel que fût le milieu d'origine. Cependant, celles obtenues à partir de gélose-terre étaient plus fraîches d'aspect.

Une nouvelle expérience, faite au sixième mois, après l'ensemencement initial, à partir des colonies maintenues sur gélose-terre, 
nous a permis d'obtenir sur Sabouraud des souches ayant tous les caractères normaux des colonies les plus fraîches qui soient, et ne manifestant aucune trace de dégénérescence pléomorphique.

Nous pouvons conclure de ceci qu'il est possible actuellement, en conservant les Dermatophytes sur de la gélose-terre, de se contenter de deux repiquages annuels pour maintenir dans le plus parfait état de conservation une collection de Dermatophytes. Il est en effet peu vraisemblable que les observations que nous avons faites sur cinq espèces caractéristiques des cinq genres de Dermatophytes que nous reconnaissons ne soient pas applicables à toutes les espèces. Ces observations nous montrent également que non seulement le sucre, mais encore la peptone, sont des substances qui favorisent le développement du pléomorphisme.

\section{VIII. - Mutation déterminée par l'emploi des milieux à base de terre}

L'observation que nous allons rapporter n'a pu être faite que sur une espèce de Dermatophyte : Langeronia soudanensis (Joyeux 1912) Vanbreuseghem 1950. Ce dermatophyte, cultivé sur Sabouraud-terre, se développe jusqu'au $25^{\circ}$ ou $30^{\circ}$ jour, suivant sa morphologie habituelle : les colonies acuminées sont d'une belle teinte rouille vif, qui rappelle fortement les colonies de $T$. ferrugineum. Mais, à partir du $25^{\circ}$ au $30^{\circ}$ jour, on voit apparaitre dans un bon nombre de souches, en un point quelconque de la surface, une petite zone blanchâtre qui se met à grandir rapidement, plus rapidement que le reste de la colonie. En huit à quinze jours, cette zone blanche est devenue d'un beau violet foncé. Le repiquage de cette partie mutée sur milieu de Sabouraud, donne la même colonie violette, et celle-ci est restée inchangée depuis six mois que nous l'observons.

L'emploi de Sabouraud-terre est indispensable pour la production du mutant violet de L. soudanensis : le milieu de conservationterre ne nous a jamais donné une seule colonie mutée. D'autre part, la présence de terre doit être considérée comme un catalyseur d'une transformation, qui peut se produire en l'absence de terre. C'est en effet sur Sabouraud que Rivalier (1) fut le premier à observer cette transformation dans une souche que nous lui avions envoyée, et nous avons pu en faire nous-même l'observation exceptionnellement.

(1) Communication personnelle. 
La mutation de $L$. soudanensis de sa forme normale à sa forme violette ne se fait pas avec une égale facilité pour toutes les souches. Notre première observation a porté sur la souche RV 4311 de notre collection: ensemencée sur Sabouraud-terre, elle produit dans $100 \%$ des cas un mutant violet. De 14 autres souches étudiées, trois nous ont donné la même mutation, tandis que les 11 autres se développaient, soit sous la forme normale, soit en se transformant progressivement en une forme blanche, que nous ne considérons pas comme un mutant, mais comme une évolution normale possible de $L$. soudanensis.

On est curieux de savoir si cette mutation de $L$. soudanensis s'accompagne d'une modification importante de sa morphologie intime. L. soudanensis, on le sait, produit sur ses hyphes principales des rameaux latéraux secondaires qui croissent en sens opposé ; il se reproduit surtout par des arthrospores qui, une fois libérées, peuvent créer dans les préparations l'image d'aleuries. Nous avons pensé que, pour observer le mieux ce mutant violet microscopiquement, nous devions avoir recours à des cultures sur lames. Toutefois, la mutation en cultures sur lames ne s'est pas produite aussi régulièrement qu'en cultures ordinaires. Nous avons pu, cependant, assister, pour ainsi dire, à la naissance du mutant violet : dans l'ensemble, celui-ci a la même morphologie que la forme normale, mais les hyphes secondaires qui croissent à rebours sont beaucoup moins nombreuses. Il s'ensuit que l'aspect buissonneux est moins marqué et que la colonie paraît plus aérée. Outre cette modification de la morphologie microscopique, nous avons observé l'apparition de pelotons mycéliens, qui peuvent former des masses arrondies de 40 à $50 \mu$, et qui sont constitués par des filaments extrêmement minces. La naissance et la constitution exacte de ces pelotons sont difficiles à saisir ; nous avions cru y voir des périthèces, en formation, mais nos observations, poussées jusque sur des cultures de 80 jours, ne nous ont pas permis de conclure dans ce sens.

On pourrait discuter sans fin sur le sens de cette mutation, et notamment se demander si ce que nous avons pu réaliser au laboratoire en petit, la nature n'est pas capable de le réaliser en grand. La ressemblance assez marquée du mutant violet de $L$. soudanensis avec d'autres. Dermatophytes conduit naturellement à rechercher l'origine de ceux-ci dans des mutations spontanées. C'est une possibilité, mais nous ne pouvons jusqu'ici tirer aucune autre conclusion. 


\section{Résumé et conclusion}

Nous avons cultivé des Dermatophytes sur des milieux de culture à base de terre : Sabouraud-terre, conservation-terre, et géloseterre, et nous avons comparé la morphologie macroscopique et microscopique des colonies obtenues sur ces milieux à celle des colonies développées sur milieu de Sabouraud. Les Dermatophytes cultivés sur milieux à base de terre produisent en grande abondance des formes de reproduction et les filaments mycéliens s'enrichissent en protoplasme. On peut également cultiver les Dermatophytes ou des produits pathologiques envahis par des Dermatophytes, - cheveux, poils, ongles, squames -, sur de la terre stérile, sans qu'il soit nécessaire d'ajouter aucune autre substance nutritive. Les colonies ainsi obtenues sont pathogènes pour l'homme et pour le cobaye. Il nous a été possible de ramener à la normale, par culture sur conservation-terre, deux souches pléomorphiques de Ctenomyces persicolor et d'Epidermophyton floccosum. La culture de Langeronia soudanensis sur Sabouraud-terre donne naissance à un mutant violet : ceci conduit à se demander si de semblables mutations ne peuvent pas se réaliser spontanément dans la nature. D'autre part, l'emploi des milieux à base de terre nous a montré qu'on pouvait conserver en parfait état, et sans aucune trace de dégénérescence pléomorphique, des Dermatophytes durant six mois, à condition d'employer le milieu gélose-terre.

Les observations que nous venons de rapporter donnent quelque vraisemblance à l'hypothèse exprimée par l'un de nous (Vanbreuseghem, 1952) et suivant laquelle les Dermatophytes vivraient leur vie normale dans la terre en attendant l'occasion propice d'un parasitisme humain ou animal. On trouve dans cette hypothèse une explication à ces «premiers cas », qui sont le point de départ des épidémies et des épizooties. Encore faudrait-il étayer cette hypothèse par l'isolement des Dermatophytes du sol. Cet isolement sera sans doute difficile à réaliser, étant donné l'abondance des moisissures dans le sol et la rapidité de leur développement sur les milieux de culture. Vanbreuseghem (1952) semble avoir résolu une partie du problème en utilisant des cheveux humains pour isoler des Dermatophytes de terre artificiellement contaminés par ces parasites. Il reste néanmoins à en faire l'application sur le terrain.

\section{BiBLIOGRAPHIE}

Ajello (L.), Grant (V. Q.), et Gutzke (M. A.). - Use of mineral oil in the maintenance of cultures of fungi pathogenic for humans. Arch. Dermat. Syph. Chicago, 1951, $63: 6,747-749$. 
Bonar (L.) et Dreyer (A. D.). - Studies on ringworm fungus with reference to public health problem. Am. Jl. Publ. Health, 1932, 22 : 9, 909-926.

Emmons (C. W.). - The isolation from soil of fungi which cause disease in Man. Trans. New-York Acad. of Sciences, 1951, ser. II, vol. 14, 51.

Gondon (M.). - Communication personnelle de L. Ajello, le 5 mars 1952.

Mandels (G. R.), Stahl (W.H.) et Levinson (H.S.). - Structural changes in wool degraded by the ringworm fungus, Microsporum gypseum, and other microorganisms. Textile Record JI., 1948, 18, 224-231.

Muende (I.) et WebB (P.). - Ringworm fungus growing as a saprophyte under natural conditions. Arch. Dermat. Syph., 1937, 36, 987-990.

Sabouraud (R.). - Les Teignes, Paris, Masson, 1910.

VANbreuseghem (R.). - Contribution à l'étude du Ctenomyces persicolor. Apport d'un cas personnel. Ann. Paras., 1949, 24 : 1-2, 124-142.

- Etude sur le Trichophyton soudanense: sa présence au Congo Belge. Création du genre Langeronia. Ann. Paras., 1950, 25 : 5-6, 493-508.

Essai de synthèse sur les Dermatophytes et le traitement des affections qu'ils déterminent. Rapport présenté à la séance du Cinquantenaire de la Société belge de dermatologie et de syphiligraphie, Bruxelles, 17 et 18 novembre 1951,46 pages.

Le cycle biologique des dermatophytes et l'épidémiologie des dermatophyties. Soc. belge de dermat. et de syphil. Sous presse in Arch. belges dermat. et syphil.

- Technique biologique pour l'isolement des Dermatophytes du sol. Ann. Soc. belge Méd. Trop. (présenté à la séance du 10 avril 1952).

- et Van Brussel (M.). - Culture de dermatophytes sur terre et sur milieux à base de terre. Soc. Biol., séance du 26 janvier 1952, scus presse in C.R. Soc. Biol., séance du 26 janvier 1952.

Réversibilité du pléomorphisme des Dermatophytes. Soc. Biol., séance du 26 janvier 1952, sous presse in C.R. Soc. Biol., séance du 23 février 1952.

- C Culture sur terre et sur milieux à base de terre, de cheveux, de poils, de squames et d'ongles infectés par des Dermatophytes. Soc. Biol., séance du 26 janvier 1952, sous presse in C.R. Soc. Biol., séance du 26 janvier 1952.

- Pouvoir pathogène des Dermatophytes cultivés sur terre. Soc. Biol., séance du 26 janvier 1952, sous presse in C.R. Soc. Biol., 23 février 1952.

- - La terre, facteur de mutation d'un dermatophyte : Langeronia soudanensis (Joyeux, 1952), Vanbreuseghem 1950. Ann. Soc. Belge Méd. Trop., 1952, 32, 1, 79-84.

- Longévité et vitalité des Dermatophytes cultivés sur milieux à base de terre. Ann. Soc. Belge Méd. Trop., 1952, 32, 2.

White (W. L.), Mandels (G. R.) et SrU (A.G.H.). - Fungi in relation to the degradation of woolen fabrics. Mycologia, 1950, 42, 199-223.

Williams (J. W.). - The habitat of Trichophyton interdigitale outside the body. Proc. Soc. Exp. Biol. and Med., 1939-1934, 31, 984-986.

(Travail effectué à l'Institut de Médecine tropicale Prince-Léopold, à Anvers. Directeur : $P^{\mathrm{r}} D^{\mathrm{r}}$ A. Dubois) 